ELUA

ISSN 2171-6692

Núm. 36, 2021, págs. 171-176

https://doi.org/10.14198/ELUA.2021.36.10

\title{
Fernández Corbacho, Analí. (2021). Aprender a leer en una lengua extranjera en educación primaria. Huelva: Universidad de Huelva. 330 Páginas
}

\author{
María-CARMEn SÁNCHEZ-VizCAÍNO \\ University of Economics, Bratislava \\ mariacarmen.vizcaino@euba.sk \\ https://orcid.org/0000-0002-4477-8557
}

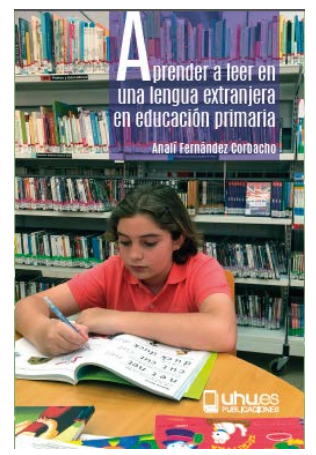

Aprender a leer en una lengua extranjera en educación primaria inicia su recorrido advirtiendo del poderoso valor que atesora la lectura, ya que es «la principal herramienta de comunicación y acceso al conocimiento» (p.23). De hecho, Fernández Corbacho señala que la alfabetización universal fue uno de los objetivos de la ONU para 2015 y de nuevo se encuentra en la Agenda 2030 para el Desarrollo Sostenible. La lectura nos acerca lo lejano justificando la inmensidad del mundo; también nos introduce en nuevos territorios al mismo tiempo que agrupa personas y culturas. En realidad, si pudiéramos leer en varias lenguas, contaríamos con un universo aún más extenso de posibilidades. Por ello, la autora, a través de una redacción exquisita y rigurosa, traza el camino que se ha de transitar para alcanzar el éxito en la lectura en una lengua extranjera (LE) en una edad temprana.

En las páginas preliminares la autora se ocupa de justificar la relevancia de la obra y especificar sus objetivos: exponer las teorías existentes y aplicables a la lectura en edad temprana; de qué destrezas o componentes específicos está compuesto el proceso lector en una LE; cuáles son los principales factores que influyen en su aprendizaje y desarrollo o la diferencia con la lectura en la lengua materna (L1). A modo de complemento, el libro también aspira a identificar los problemas lectores en inglés como LE entre aprendices españoles de Educación Primaria. En definitiva, Fernández Corbacho pretende hallar evidencias para poder determinar lo antes posible a estudiantes con dificultades lectoras $\mathrm{y}$, de forma más extensiva, que el alumnado en una edad temprana alcance un buen nivel de lectura en cualquiera de las lenguas que

Para citar esta reseña: Sánchez-Vizcaíno, M.-C. (2021). Fernández Corbacho, A. (2021). Aprender a leer en una lengua extranjera en educación primaria. Huelva: Universidad de Huelva. 330 páginas. ELUA, (36), 171-176. https://doi.org/10.14198/ELUA.2021.36.10

(C) 2021 María-Carmen Sánchez-Vizcaíno

Este trabajo está sujeto a una licencia de Reconocimiento 4.0 Internacional de

Creative Commons (CC BY 4.0) 
aprenda. Seguidamente, ofrece un breve resumen de cada uno de los seis capítulos que componen el trabajo. De aquí se trasluce un recorrido sistemático y bien fundamentado partiendo de las teorías más relevantes sobre el tema para presentar un estudio empírico enriquecido con tablas y figuras que desembocará en un marco general para una intervención lectora adecuada.

Es una obra que se recomienda para maestras, maestros, madres y padres, conforme apunta en el prólogo la Dra. M. Carmen Fonseca Mora. Además, también será de utilidad para estudiantes graduados y formadores del profesorado, así como para quien desee adquirir una perspectiva actual en torno a las principales aportaciones teóricas y prácticas sobre la enseñanza del proceso lector en lengua extranjera en edad temprana.

En el primer capítulo, Fernández Corbacho desgrana las teorías más representativas que explican la adquisición y desarrollo de la destreza lectora. De ahí emana la importancia de la lectura a la hora de aprender una lengua y las características de un buen lector. En este sentido el entorno social y afectivo del niño o la niña desempeña un papel relevante, ya que los primeros contactos con los libros y las historias de mano de los adultos son determinantes para la evolución posterior en dicho ámbito. Un buen lector «realiza una lectura fluida y comprensiva de un texto» (p. 33), pero para ello «ha de ser un agente activo capaz de resolver problemas y usar su conocimiento y experiencia previos» (p. 33). A continuación, se define el proceso lector a la vez que se presentan los componentes involucrados en el mismo. En ese camino hacia la eficiencia lectora el lector principiante recorre varias fases preparatorias entre las que destaca la decodificación. Por su parte, en la adquisición y desarrollo de la destreza lectora es crucial conocer las habilidades prelectoras (motivación por lo impreso, conciencia gráfica, conciencia fonológica, conocimiento del alfabeto, vocabulario y destrezas narrativas), ya que son determinantes para llegar a ser un buen lector.

El capítulo continúa con una exposición detallada de los diferentes modelos que explican cómo aprendemos a leer y la evolución y características de estas etapas. Es de destacar que recoja las últimas tendencias, como los trabajos de Wagner et al. (2006), quienes introducen el modelo de lectoescritura emergente en el que destacan la sensibilidad fonológica, el lenguaje oral y la conciencia gráfica, en cuanto a habilidades clave en niños prelectores; al igual que el modelo multivariado de Konold et al. (1999, 2003), que parte de la integración del reconocimiento de palabras y la comprensión oral para posibilitar la comprensión lectora. La sección culmina presentando los desafíos que pueden impedir un buen desarrollo lector. En este sentido, si el aprendiz no aprende a decodificar bien, no podrá pasar a la siguiente etapa y, por tanto, tendrá consecuencias en su rendimiento escolar a la vez que en su autoestima porque se verá incapaz de poder leer; al contrario que sus compañeros. Estos problemas de comprensión suelen ocurrir en segundo de primaria, ya que los textos poseen una mayor complejidad. Dicho fenómeno puede aparecer por deficiencias en el desarrollo de la lengua oral. De hecho, Fernández Corbacho afirma que «para tener éxito en la escuela, y en el mundo actual, es imperativo convertirse en un buen lector en tercer curso o alrededor de los 8 años» (p. 59). Por consiguiente, la autora aboga por una detección temprana de las dificultades lectoras para prevenir situaciones problemáticas en el futuro.

El segundo capítulo gira en torno a la lectura en LE en donde se exponen de forma detallada los componentes lectores, además de las transferencias e interrelaciones que se establecen entre la L1 y la LE. Los componentes lectores, presentes tanto en la L1 como en la segunda lengua (L2), serán clave para comprender el proceso lector y, por ende, para tomar decisiones a la hora de abordar la enseñanza de la lectura. No obstante, también aparecen 
variaciones entre la L1 y la L2 que dependen de «factores como las similitudes y diferencias entre ambas lenguas, o el tipo de contacto con la LE» (p. 63). Partiendo de la premisa de que la lectura beneficia al manejo global de la LE (Day y Bamford, 1998; Krashen, 1993, 2004; Rodrigo, 2019), se ha de considerar su aprendizaje en una LE un proceso sumamente complejo en el que intervienen variados factores: «la lengua materna, la cultura nativa, el contexto de instrucción, el contacto con la LE, las diferencias individuales, etc.» (p. 64).

En cuanto a la interrelación entre la L1 y la LE se puede destacar que, por lo general, un buen lector en L1 también lo es en LE. Asimismo, «el sistema fonético de la L1 del aprendiz puede influir en algunos aspectos del aprendizaje de la L2, como son la articulación, la discriminación auditiva o la lectura oral» (p. 71) y «al leer en LE transferimos los conocimientos y estrategias adquiridas en la lengua materna» (p. 76). A continuación, Fernández Corbacho se centra en los aspectos problemáticos para aprendices hispanohablantes de inglés. En particular, se han de tener presente las semejanzas y diferencias entre ambas lenguas: la decodificación, la lengua hablada, además de otros aspectos gramaticales y sintácticos. El capítulo cierra con las características del buen lector de una LE. En este punto la autora señala que leer en una LE en muchos casos «supone un uso más consciente de habilidades y estrategias lectoras, de procesamiento cognitivo y lingüístico, anticipación al texto, comprensión o de construcción del significado» (p. 94).

El capítulo tercero vertebra la enseñanza de la destreza lectora en el que se revisan los enfoques metodológicos empleados hasta la fecha y que continúan aplicándose de acuerdo con el concepto de la lectura que considere el enseñante. Fernández Corbacho apunta que tradicionalmente no se ha llevado a cabo una enseñanza correcta de la lectura en LE. Además, afirma que es imprescindible trabajar con los componentes de la lectura (conciencia fonológica, código alfabético, fluidez, vocabulario y comprensión), y la lengua oral; por lo que resulta indispensable la exposición a la lengua hablada, es decir, a la lengua meta. Por lo tanto, primero se ha de trabajar la lengua oral antes de enseñar a leer en LE. También es relevante la actuación del docente y la participación de las familias para estimular al niño pre-/ lector. Más adelante se detallan los enfoques metodológicos para la enseñanza de la lectura donde aparecen los sintéticos, los analíticos y otros métodos. Con respecto a las implicaciones pedagógicas de estudios sobre la enseñanza de la lectura en una LE a escolares, la autora sugiere «adoptar enfoques flexibles que se puedan adaptar a las circunstancias y necesidades de los estudiantes» (p.133). Es interesante señalar la importancia de las variables afectivas en la enseñanza de la lectura, como la actitud o la motivación. El docente ha trasladar al aprendiz la diversión que esconde la lectura, ya que «si hay frustración, no hay motivación» (p. 136). En este sentido la familia ocupa un papel primordial en la motivación de los lectores.

A continuación, Fernández Corbacho introduce las competencias (académica, digital, evaluadora) de los buenos formadores, quienes han de «conocer el proceso lector, ser conscientes de la importancia de las diferencias individuales, de los diferentes conocimientos, experiencias y niveles de motivación de los aprendices» (p. 137). Además, se alude a la relevancia de su formación en este campo, tanto en sus inicios como a lo largo de su carrera profesional. El capítulo se cierra con las pruebas de detección de problemas de lectura (DIBELS-6, IDEL-7, EGRA o DRA Word Analysis) en LE en niveles iniciales, es decir, con la trascendencia de identificar y realizar un adecuado diagnóstico de los problemas lectores. Por ello, el profesorado necesita ser competente; con lo que es necesario que evalúe a sus aprendices para detectar dichos problemas y así poder intervenir de forma adecuada. 
El capítulo cuatro introduce el estudio empírico que ha llevado a cabo la autora, los objetivos de la misma y el método de investigación utilizado. Primero, Fernández Corbacho plantea el estudio donde apunta que el momento idóneo para iniciar la lectura en LE es cuando el lector ya ha logrado cierto grado de consolidación en la lectura en L1, es decir, cuando ya domina la destreza decodificadora. También indica la relevancia de tener en cuenta factores lingüísticos (dominar los cinco componentes de la destreza lectora), cognitivos (memoria de trabajo) y sociales (contexto cultural, familiar y escolar del lector). A partir de aquí fundamenta su investigación advirtiendo de la necesidad de contar con un estudio sobre el rendimiento lector de aprendices españoles de inglés LE. De esta forma, detalla el objetivo principal del estudio: establecer relaciones entre la habilidad lectora en LE y la destreza lectora en la L1, factores cognitivos (memoria de trabajo, comprensión verbal y velocidad de procesamiento) y la situación sociocultural del aprendiz. Asimismo, expone una serie de objetivos más concretos.

Posteriormente, el siguiente apartado del capítulo es la descripción del método. En esta investigación participaron 47 alumnos de $2^{\circ}$ de Educación Primaria de un centro educativo situado en una zona de clase socioeconómica vulnerable. Los instrumentos de recogida de datos fueron la prueba de lectura en español y en inglés (EGRA), el test neuro-psicológico (WISC-IV), un cuestionario sociocultural y entrevistas grupales e individuales. Finalmente, el procedimiento se llevó a cabo en las siguientes etapas: administración de las pruebas al principio del trimestre y realización del cuestionario sociocultural, además de las entrevistas (individuales y grupales).

El capítulo cinco recoge los resultados del estudio empírico, expuestos de forma detallada de acuerdo con variables lingüísticas, cognitivas y sociales. En primer lugar, se describe la situación sociocultural de los participantes en la investigación en la que destaca la diversidad cultural y el hecho de que en gran parte de las familias se lee en casa. Además, entre ellas, las familias multilingües cuentan con un nivel educativo superior; a pesar de ello, por lo general, ninguna se implica en la vida escolar. A continuación, se exponen los resultados según los objetivos propuestos. El primero de ellos pretendía identificar las áreas problemáticas de la lectura en L1 y LE donde se confirmó que los resultados de las pruebas fueron peores en L1 que en LE y que la mayoría de los aprendices presentaron dificultades. El segundo objetivo buscaba la vinculación entre la destreza lectora en L1 y LE. Las variables con mayor correlación fueron la conciencia fonológica, seguida de la decodificación y la fluidez. Después, se aspiraba a reducir el número de variables lectoras para facilitar su análisis. A tal efecto, la autora se apoyó en un estudio estadístico que detalla junto con tablas y gráficos. Luego se perseguía averiguar la relación entre destrezas lectoras y variables cognitivas, donde la memoria de trabajo fue la variable que presentó correlaciones más altas con las destrezas lectoras.

El siguiente objetivo proponía establecer la relación entre la destreza lectora y aspectos socioculturales. De ahí se dedujo que el contacto familiar con otra lengua y el nivel educativo materno presentaron relaciones significativas con las destrezas lectoras, tanto en L1 como en L2, especialmente en las destrezas de fluidez y decodificación en español L1. El objetivo seis pretendía definir un perfil de estudiante con dificultades lectoras en LE. Los niños y niñas con problemas lectores no tenían contacto con otra lengua en casa y el nivel educativo materno era limitado. Los cálculos estadísticos ayudaron a alcanzar el objetivo siete: calcular la probabilidad de que los aprendices tuvieran problemas de rendimiento 
académico en la LE y la L1. Finalmente, el último de los objetivos aspiraba a proponer una herramienta de evaluación y diagnóstico de la destreza lectora válida para L1 y LE. Para ello se examinaron los resultados obtenidos y se consideraron la opinión y las propuestas de mejora de la herramienta de los administradores de las pruebas EGRA.

Finalmente, la obra se cierra con la discusión de los resultados alcanzados, así como con la exposición de las conclusiones y las implicaciones pedagógicas del estudio empírico en donde se detalla un marco general para una intervención lectora adecuada. En primer lugar, de la situación sociocultural de los aprendices involucrados se desprende la escasa implicación de las familias en la formación escolar de sus hijos. En cuanto a la destreza lectora en L1 y LE, las áreas problemáticas en L1 parecen ser la comprensión lectora, la conciencia fonológica y la comprensión oral. Este último detalle es preocupante dado que los aprendices no entienden cuando se les lee en su lengua materna. Con respecto a las áreas problemáticas en LE, se advierte de una deficiencia en comprensión lectora porque tienen un nivel de competencia en LE escaso.

Por lo tanto, el aprendiz ha de poseer un dominio básico de la LE para comprender un texto escrito. El reducido vocabulario que manejan o la limitada formación académica de las madres también influyen de forma negativa en el desarrollo de las destrezas lectoras. Es más, se percibe una destreza lectora pobre en todas sus facetas: fonológica, ortográfica y comprensiva. El grupo investigado se caracteriza por su disparidad con respecto a las habilidades lectoras tempranas y que se encuentran sin desarrollar de manera completa. Por consiguiente, el profesorado ha de estar atento a las siguientes señales que indican que el aprendiz podría tener problemas lectores: vocabulario oral en la LE escaso; no codifica ni recodifica bien en la LE; su desarrollo lector en la L1 no está completado o procede de un entorno sociocultural vulnerable. Por su parte, la relación entre las habilidades lectoras en L1 y LE nos indica que es necesario un conocimiento básico de la lengua meta. Además, para evaluar la comprensión lectora de un aprendiz español de inglés LE hay que medir la fluidez lectora, las destrezas fonológicas y la comprensión.

Asimismo, la relación de la destreza lectora y variables cognitivas y socioculturales indica que la memoria de trabajo, el contacto con otras lenguas y el nivel educativo de los progenitores están relacionados. Si se ahonda en la descripción de los perfiles lectores identificados, cuando haya desafíos lectores en L1 los habrá igualmente en LE. Por regla general, los aprendices españoles con problemas en la lectura en LE tienen problemas de decodificación y fluidez, problemas con las destrezas lectoras en L1, deficiencias en la memoria de trabajo y en la velocidad de procesamiento, y su madre es de nacionalidad española, sin estudios o graduado escolar. A continuación, la autora presenta las conclusiones en donde afirma que la destreza en LE dependerá del conocimiento de la lengua meta, pero también de la fase de desarrollo lector en la L1 y algunas variables cognitivas como la memoria de trabajo y la velocidad de procesamiento. Además, se describe al alumno con problemas lectores en la LE: tiene problemas con la lengua materna, de decodificación y fluidez en la LE, y no destaca en las destrezas fonológicas; tiene ciertas deficiencias en la memoria de trabajo y la velocidad de procesamiento, y la madre, de nacionalidad española, cuenta con un nivel de estudios limitado.

El libro se cierra con la implicación pedagógica del estudio: un marco general para una intervención lectora adecuada. De ello se desprenden una serie de premisas como que hay que ser lectores en la L1 antes de introducir la lectura en la LE. Por su parte, el profesorado 
ha de estar formado y motivado para enseñar destrezas lectoras, pero también ha de detectar los posibles problemas para poder intervenir. Asimismo, se necesita el apoyo familiar y del centro escolar. Ahora bien, es necesaria una evaluación completa de la capacidad lectora en función de las destrezas fonológicas, la fluidez lectora y la comprensión. En este sentido, la autora propone una herramienta de evaluación de la destreza lectora en LE y L1 donde se incluyen pruebas de conciencia fonológica tanto en L1 y en LE; pruebas de decodificación y otras para detectar el conocimiento general de la lengua. Por último, se describen las limitaciones del estudio y las futuras líneas de investigación.

En suma, Aprender a leer en una lengua extranjera en educación primaria aporta las claves necesarias para comprender la importancia de las diferentes variables que entran en juego para el éxito en la lectura en una LE en una edad temprana. Su autora cumple ampliamente los objetivos establecidos al comienzo de la obra. No solo aduce argumentos sólidos para la comprensión del proceso lector, sino que también nos orienta acerca del procedimiento que hay detrás de un estudio sobre el aprendizaje de la lectura en inglés como lengua extranjera. Su diferenciación radica en la implicación pedagógica, ya que se incluyen interesantes propuestas para una intervención lectora temprana que maestras, maestros, madres y padres han de conocer. Aprender a leer es un proceso que comienza incluso antes de tener consciencia de este. Por ello, si guiamos a nuestros niños y niñas de forma adecuada podrán, a partir del momento conveniente, empezar a disfrutar del maravilloso mundo que se desprende al abrir la página de un libro.

\section{REFERENCIAS}

Day, R. y Park J.-S. (1998). Extensive Reading in the Second Language Classroom. Nueva York: Cambridge University Press.

Konold, T.R., Juel, C. y McKinnom, M. (1999). Building an integrated model of early reading acquisition (Vol, 1-103). Michigan: CIERA. Universidad de Michigan.

Konold, T.R., Juel, C., McKinnom, M. y Deffes, R. (2003). A multivariate model of early reading acquisition. Applied psycholinguistics, 24 (01), 89.

Krashen, S. (1993). The Case for Free Voluntary Reading. The Canadian Modern Language Review 50 (1): 72-82.

Krashen, S. (2004). The Power of Reading: Insights from the Research. Portsmouth, Nueva Hampshire: Heinemann.

Rodrigo, V. (2019). La comprensión lectora en la enseñanza del español LE/L2. Londres / Nueva York: Routledge. 